\title{
Sobre os procedimentos de cálculo de conectores parafusos em pilares tubulares de aço preenchidos com concreto
}

\author{
Lucas Ribeiro dos Santos ${ }^{1 *}$, Rodrigo Barreto Caldas ${ }^{2}$, Ricardo Hallal Fakury², \\ Francisco Carlos Rodrigues ${ }^{2}$ e Hermano de Sousa Cardoso ${ }^{2}$ \\ 1*, 2 Universidade Federal de Minas Gerais, Programa de Pós-Graduação em \\ Engenharia de Estruturas, Av. Antônio Carlos, 6627 - Escola de Engenharia, \\ Bloco I - 40 andar - Sala 4215 - Pampulha - Belo Horizonte - MG. \\ e-mail: luccasrsantos@gmail.com,rbcaldas@gmail.com, fakury@dees.ufmg.br, \\ francisco@dees.ufmg.br, hermanocardoso@yahoo.com.br
}

\section{About the design procedures of bolts connectors in concrete-filled steel tubular columns}

\begin{abstract}
Resumo
Neste trabalho foram realizados ensaios de cisalhamento na região de introdução de força dos pilares tubulares circulares de aço preenchidos com concreto para a avaliação do comportamento de conectores parafusos. A utilização desses conectores em pilares mistos tubulares preenchidos tem se demonstrado viável, uma vez que são de fácil instalação e de baixo custo. Os resultados dos ensaios foram analisados avaliando-se a rigidez, a força máxima e os modos de falha e comparados com aqueles fornecidos pelos procedimentos de cálculo encontrados na literatura, dentre eles, o da norma brasileira ABNT NBR 16239:2013. Foi possível constatar que os procedimentos de cálculo forneceram resultados significativamente inferiores aos dos ensaios para a capacidade resistente dos conectores, sendo que os menores valores foram os da ABNT NBR 16239:2013.
\end{abstract}

Palavras-chave: conectores de cisalhamento, conectores parafusos, pilares mistos preenchidos com concreto, ensaios de cisalhamento.

\begin{abstract}
In this study, push tests were performed in the load transfer region of concrete-filled steel tube for the evaluation the behavior of bolt connectors. The usage of these devices in composite columns has been regarded as a feasible design option, due to its easiness to be installed and for being a low-cost technology. The results tests were analyzed through the evaluation of the stiffness, ultimate load and failure modes. These results were also compared with those provided by the calculation procedures found in the literature, among them, the Brazilian standard ABNT NBR 16239:2013. It was possible to verify that the calculation procedures gave results significantly inferior to the tests for the resistant capacity of the connectors and that the lower values were those of ABNT NBR 16239: 2013.
\end{abstract}

Keywords: shear connectors, bolts connectors, composite column, push-test.

* autor correspondente 


\section{Introdução}

\subsection{Considerações Gerais}

Nos pilares mistos é essencial que haja interação entre os componentes perfil de aço e concreto. Algumas vezes a aderência natural não é capaz de proporcionar a interação adequada, tornando necessária a utilização de dispositivos mecânicos, como os conectores de cisalhamento, para a transferência de forças entre os componentes.

Estudos recentes na Europa, entre os quais os de Starossek e Falah (2008), Athira (2016) e Younes et al. (2016), mostraram que os conectores de cisalhamento constituídos por parafusos (chamados aqui de conectores parafusos) são uma solução interessante para a ligação entre o tubo de aço e o núcleo de concreto nos pilares mistos preenchidos com concreto (PMPC). No Brasil, esses dispositivos têm sido estudados por alguns pesquisadores, com destaque para os trabalhos de Almeida (2012), Cardoso (2014), Santos et al. (2016a, 2016b), Santos (2017) e Ribeiro Neto e Sarmanho (2017). Também vale ressaltar que na norma brasileira ABNT NBR 16239:2013 foi introduzido o modelo de cálculo desses conectores nos PMPC.

Neste trabalho são apresentados ensaios de cisalhamento na região de introdução de força dos pilares tubulares circulares de aço preenchidos com concreto para a avaliação do comportamento estrutural de conectores parafusos. Os resultados desses ensaios foram analisados avaliando-se a rigidez, a força máxima e os modos de falha e, ainda, comparados com os resultados fornecidos por modelos de cálculo encontrados na literatura, dentre eles, o prescrito na ABNT NBR 16239:2013.

\subsection{Caracterização dos Conectores}

Considerando-se a rigidez inicial da curva força versus deslizamento entre o aço e o concreto, os conectores podem ser classificados como rígidos ou flexíveis (Almeida, 2012). Nas Figuras 1a e 1c têm-se exemplos de curvas força versus deslizamento obtidas com conectores rígidos, e nas Figuras 1 b e $1 d$, com conectores flexíveis.

Segundo a norma EN 1994-1-1:2004, o conector pode ser tomado como dúctil se a capacidade característica de deslizamento $\left(\delta_{u k}\right)$, igual ao deslizamento $\left(\delta_{u}\right)$ reduzido em $10 \%$, for maior ou igual a $6 \mathrm{~mm}$. 
O deslizamento $\left(\delta_{u}\right)$ é medido na parte descendente da curva, correspondente ao nível da força resistente característica $\left(P_{R k}\right)$, conforme se vê nas Figura 1e e 1f.

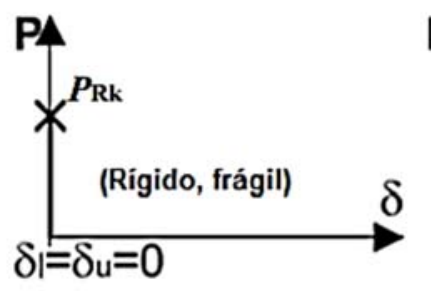

$\delta \mathrm{u}=0$

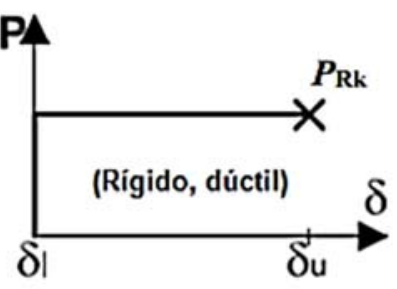

$\delta u>0$

(a)

(c)

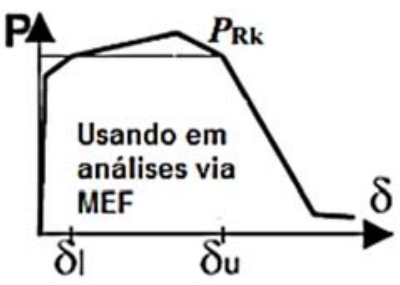

(e)
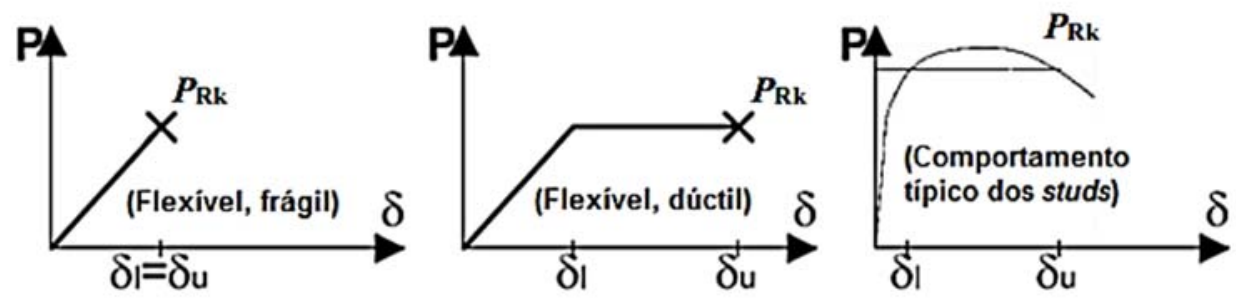

$\delta u>0$

$\delta u>0$

(b)

(d)

(f)

Figura 1 - Classificação dos conectores: (a) rígido e frágil; (b) flexível e frágil; (c) rígido e dúctil; (d) flexível e dúctil; (e) curva típica para análise via MEF; (f) comportamento típico de conectores studs (adaptado de Bartschi, 2005)

Almeida (2012) define que os conectores que possuem rigidez secante inferior ou igual a $200 \mathrm{kN} / \mathrm{mm}$ podem ser classificados como flexíveis, e os demais, como rígidos.

\subsection{Procedimentos analíticos de cálculo}

\subsubsection{Considerações gerais}

A única norma de projeto que contém um procedimento para cálculo de conectores parafusos em pilares mistos preenchidos com concreto é a brasileira ABNT NBR 16239:2013. Na literatura científica, merecem destaque os procedimentos de Starossek e Falah (2008) e Van-Long et al. (2015). Nos subitens seguintes esses procedimentos serão apresentados sucintamente.

\subsubsection{Procedimento da ABNT NBR 16239:2013}

O procedimento de cálculo da norma brasileira ABNT NBR 16239:2013 possui expressões que cobrem os três modos de falha possíveis para os conectores parafusos: 
esmagamento do concreto; cisalhamento do parafuso; e, esmagamento da parede do tubo. Assim, a força resistente de cálculo dos conectores $\left(V_{R d}\right)$ corresponde ao menor valor entre os seguintes:

$$
\begin{gathered}
V_{R d}=l_{b} \emptyset_{b} \sigma_{c, R d, N B R} \leq 5 \emptyset_{b}{ }^{2} \sigma_{c, R d} \\
V_{R d}=0,4 \pi \frac{\emptyset_{b}{ }^{2} f_{u b}}{\gamma_{a 2}} \leq 2,4 \emptyset_{b} t \frac{f_{u}}{\gamma_{a 2}}
\end{gathered}
$$

onde $l_{b}$ e $\emptyset_{b}$ são o comprimento líquido (descontando-se a espessura do tubo de aço) e o diâmetro dos parafusos, respectivamente, $t$ é a espessura da parede do tubo, $f_{u}$ e $f_{u b}$ são as resistências à ruptura do aço do tubo e do parafuso, respectivamente, e $\gamma_{a 2}$ é o coeficiente de ponderação da resistência do aço, igual a 1,35. Na Eq. (1), $\sigma_{c, R d, N B R}$ é a tensão de compressão resistente de cálculo do concreto, dada por:

$$
\sigma_{c, R d, N B R}=\frac{f_{c k}}{\gamma_{c} \gamma_{n}} \sqrt{\frac{A_{2}}{A_{1}}} \leq f_{c k}
$$

onde $A_{1}$ é a área de concreto carregada (área de contato), $A_{2}$ é a área da seção transversal do concreto, $f_{c k}$ é a resistência característica à compressão do concreto e $\gamma_{c}$ e $\gamma_{n}$ são coeficientes de ponderação da resistência e de comportamento do concreto, respectivamente, ambos iguais a 1,40. Conforme a ABNT NBR 16239:2013, a relação $A_{2} / A_{1}$ deve ser tomada como igual a 4.

Nesse procedimento de cálculo, é fácil constatar que a Eq. (1) prevê o esmagamento do concreto e, a Eq. (2), simultaneamente o cisalhamento do conector parafuso e o esmagamento da parede do tubo.

\subsubsection{Procedimento de Starossek e Falah (2008)}

No procedimento de Starossek e Falah (2008) são previstas as falhas dos conectores parafusos por esmagamento do concreto e cisalhamento do parafuso, conforme, respectivamente, as seguintes expressões para a força resistente de cálculo: 


$$
\begin{gathered}
V_{R d}=\frac{A_{1} f_{c k}}{\gamma_{c}} \sqrt{\frac{A_{c 1}}{A_{1}}} \\
V_{R d}=0,8 \pi \frac{\emptyset_{b}{ }^{2} f_{u b}}{4 \gamma_{v}}
\end{gathered}
$$

onde $\gamma_{v}$ é um coeficiente de ponderação da resistência do parafuso, igual a 1,25. O valor de $A_{c 1}$ pode ser tomado como igual a:

$$
A_{c 1}=2,25 l_{b} \emptyset_{b}
$$

\subsubsection{Procedimento de Van-Long et al. (2015)}

No procedimento de Van-Long et al. (2015), da mesma forma que Starossek e Falah (2008), são previstas as falhas por esmagamento do concreto e cisalhamento do parafuso, cujas forças resistentes de cálculo são dadas, respectivamente, por:

$$
\begin{gathered}
V_{R d}=2,5 \emptyset_{b}{ }^{2} \sigma_{c, R d, E N V} \\
V_{R d}=\alpha_{v} \frac{A_{b} f_{u b}}{\gamma_{v}}
\end{gathered}
$$

A Eq. (8) é proveniente da norma europeia EN 1993-1-8:2004 e, segundo essa norma, para o plano de cisalhamento passando na parte não roscada do fuste, $\alpha_{v}$ é igual a 0,6, $f_{u b}$ é a resistência à ruptura do aço do parafuso, $A_{b}$ é a área da seção transversal do fuste e $\gamma_{v}$ é o coeficiente de ponderação da resistência do parafuso, igual a 1,25.

Esse procedimento analítico foi desenvolvido exclusivamente para obter a capacidade resistente relacionada à falha no núcleo de concreto com parafusos passantes (contínuos ao longo do diâmetro do tubo). Para levar em conta o confinamento do concreto, na região carregada pelos conectores, os autores adotaram a equação recomendada pela norma europeia EN 1994-1-1:2004 para definir o valor da tensão de compressão resistente de cálculo do concreto, conforme segue:

$$
\sigma_{c, R d, E N V}=f_{c d}\left(1+n_{c L} \frac{t}{D} \frac{f_{y}}{f_{c k}}\right) \sqrt{\frac{A_{c}}{A_{1}}} \leq \frac{A_{c} f_{c d}}{A_{1}} \leq f_{y d}
$$


onde $n_{C L}$ é o fator de confinamento, igual a 4,9 para seções circulares, $A_{1}$ é a área de concreto carregada abaixo do fuste do parafuso (área de contato), $A_{c}$ é a área da seção transversal do núcleo de concreto onde estão instalados os conectores, $f_{c d}$ é a resistência de cálculo à compressão do concreto não-confinado, $f_{y d}$ é a resistência ao escoamento de cálculo do tubo de aço e $f_{c k}$ é a resistência característica do concreto não-confinado. A razão $A_{c} / A_{1}$ deve ser tomada com valor inferior ou igual a 20.

\section{Análise Experimental}

\subsection{Descrição Geral dos Ensaios}

O ensaio de cisalhamento padrão - standard push-test - prescrito na norma EN 1994-11:2004 é indicado para vigas mistas com lajes de espessura uniforme ou com mísulas. Para outros casos, ensaios específicos devem ser utilizados. Neste estudo foi utilizado o método de ensaio desenvolvido por Cardoso et al. (2016) para caracterizar o comportamento de conectores sujeitos ao cisalhamento nos PMPC.

Duas séries foram testadas e analisadas neste trabalho, as séries E e F, representadas nas Figuras 2 e 3, respectivamente. Os modelos da série E possuíam altura de $1.000 \mathrm{~mm}$ com conectores parafusos instalados em dois níveis, com o primeiro nível na altura de $585 \mathrm{~mm}$ e o segundo nível na altura de $700 \mathrm{~mm}$, conforme a Figura 2. Nos modelos da série $F$, quatro conectores foram instalados em um único nível na altura de $700 \mathrm{~mm}$, como se observa na Figura 3. Nota-se que essas duas séries se distinguem entre si pela disposição dos conectores em um nível ou dois níveis, e foram assim idealizadas com o objetivo de avaliar as possíveis configurações de aplicação dos conectores, estudando a influência na capacidade resistente desses dispositivos nas duas configurações distintas. Para minimizar a transferência de forças por aderência e atrito, foram aplicadas pintura e cera desmoldante na superfície interna dos tubos. Durante o ensaio, a força foi aplicada gradualmente com o auxílio de um atuador hidráulico MTS sobre a chapa de topo que, por sua vez, estava apoiada sobre o tubo de aço (Figuras 2, 3 e 4). 

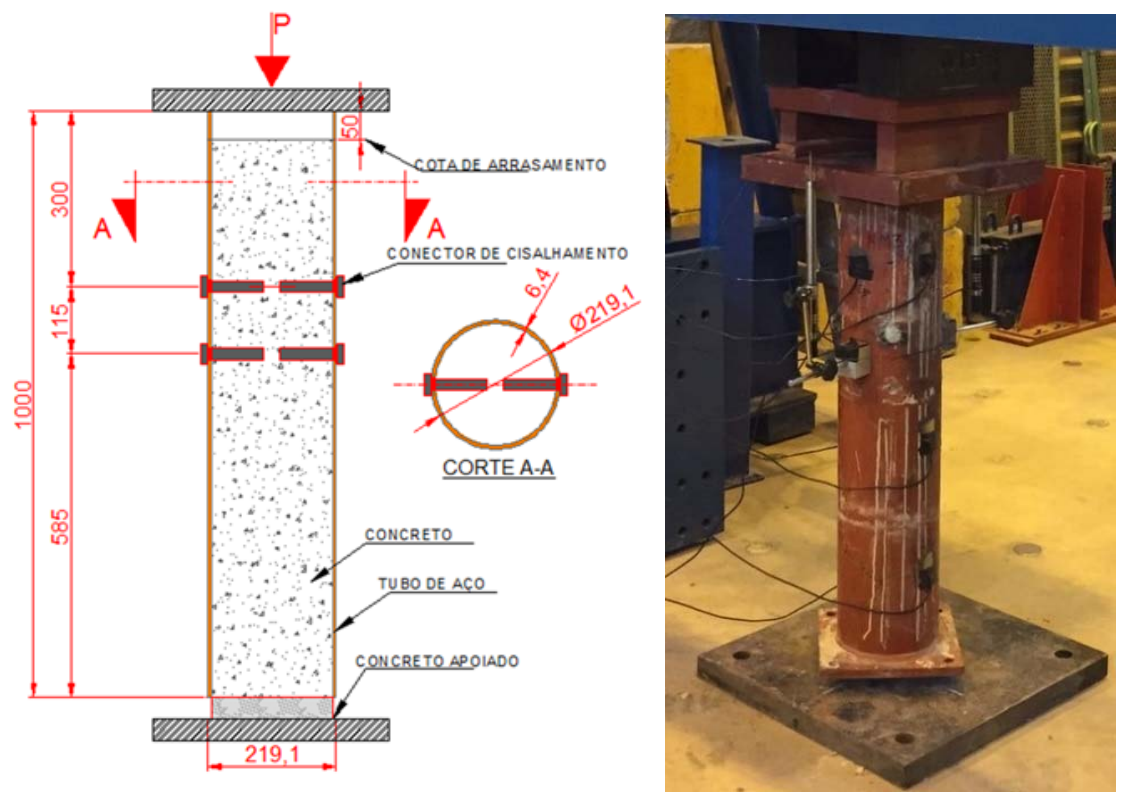

Figura 2 - série E: geometria dos modelos (dimensões em $\mathrm{mm}$ ) e imagem do ensaio
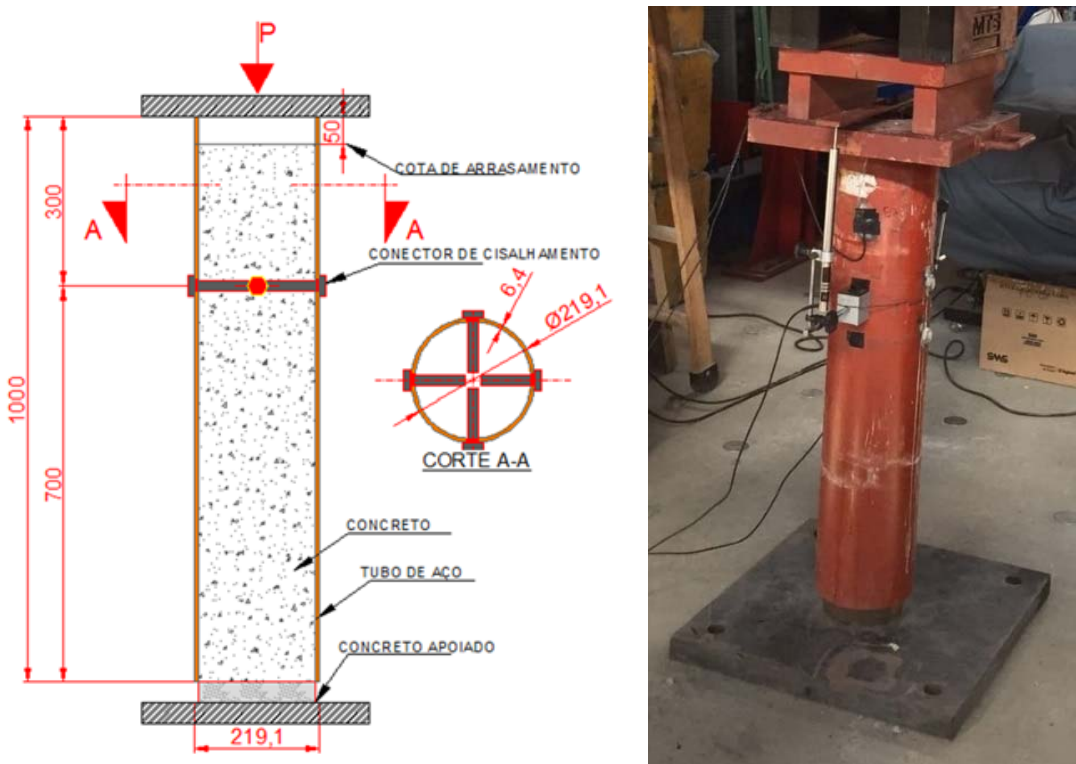

Figura 3 - série F: geometria dos modelos (dimensões em $\mathrm{mm}$ ) e imagem do ensaio

Entre o núcleo de concreto e a chapa de topo havia uma folga de $50 \mathrm{~mm}$ garantindo que a força fosse aplicada somente no tubo de aço. Na base, apenas o núcleo de concreto foi apoiado com uma chapa de aço circular com diâmetro inferior ao diâmetro interno do tubo. Dessa forma, tem-se o cisalhamento na interface de contato entre os componentes tubo de aço e núcleo de concreto solicitando os conectores.

Os deslizamentos relativos entre o tubo de aço e o núcleo de concreto foram obtidos por meio de meio de transdutores de deslocamentos (DTs) verticais. Na Figura 4 
observa-se que os DTs foram apoiados por uma base magnética no nível médio dos conectores e nivelados verticalmente até a chapa auxiliar fixada no topo do núcleo de concreto. A utilização dos DTs fixados lateralmente facilita a leitura do deslizamento relativo entre o tubo de aço e núcleo de concreto, eliminando possíveis interferências devidas às imperfeições (Cardoso et al., 2016).

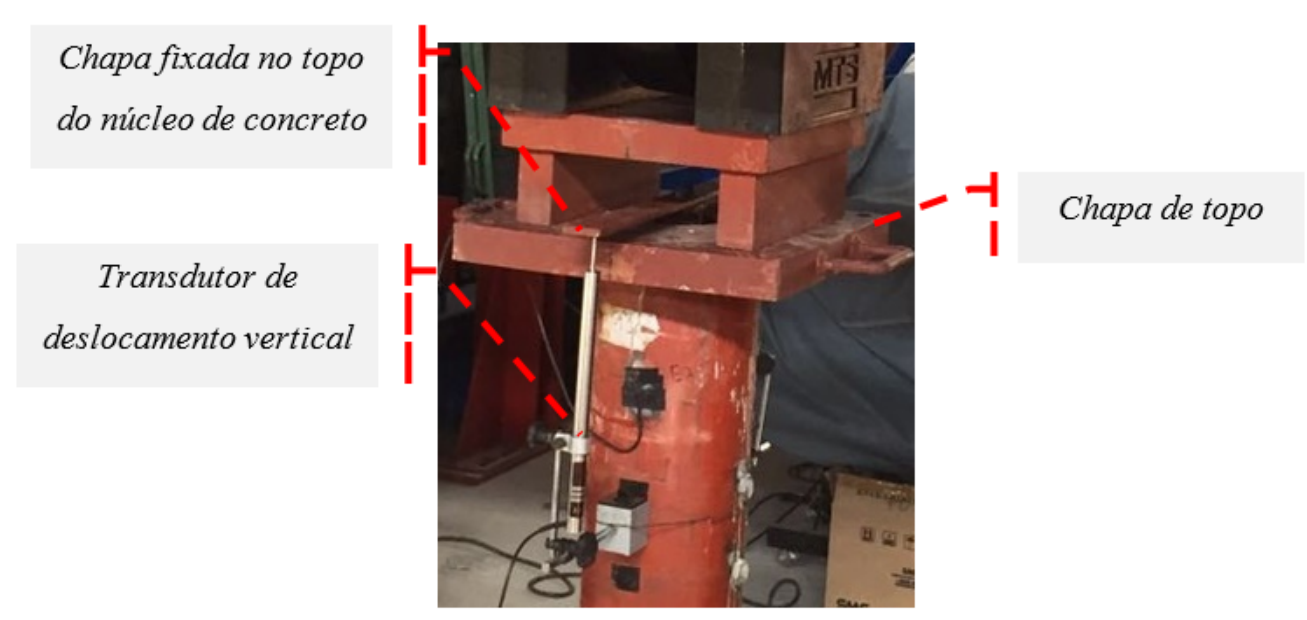

Figura 4 - Posição dos transdutores de deslocamentos durante os ensaios

Os perfis tubulares das séries $\mathrm{E}$ e $\mathrm{F}$ foram fabricados pela Vallourec Tubos do Brasil com aço VMB 350 e dimensões nominais de 219,1 $\mathrm{mm}$ e 6,4 $\mathrm{mm}$ para o diâmetro externo $(D)$ e espessura $(t)$, respectivamente. Para os conectores foram utilizados parafusos de alta resistência mecânica ASTM A325, fabricados pela CISER, com 19,05 mm (3/4") e 95,25 $\mathrm{mm}\left(3^{3} / 4^{\prime \prime}\right)$ de diâmetro $\left(\emptyset_{b}\right)$ e comprimento nominal $\left(l_{b}\right)$, respectivamente.

As resistências ao escoamento e à ruptura do aço do tubo foram obtidas por meio de ensaios feitos pelo fabricante e encontram-se na Tabela 1. Nessa mesma tabela são mostrados também os valores das resistências ao escoamento e à ruptura do aço dos parafusos. Para os parafusos, somente a resistência a ruptura foi proveniente de ensaios de caracterização, sendo a resistência ao escoamento obtida por interpolação linear a partir da resistência à ruptura, conforme a norma ASTM A325-10 1 . Para o módulo de elasticidade $(E)$ e o coeficiente Poisson $(v)$ desses produtos foram também considerados, neste estudo, os valores nominais, iguais a $200.000 \mathrm{MPa}$ e 0,3, respectivamente. 
Tabela 1 - Propriedades mecânicas dos tubos de aço e parafusos

\begin{tabular}{|c|c|c|}
\hline \multirow{2}{*}{ Material } & $\boldsymbol{f}_{\boldsymbol{y}}$ & $\boldsymbol{f}_{u}$ \\
\cline { 2 - 3 } & {$[\mathrm{MPa}]$} & {$[\mathrm{MPa}]$} \\
\hline Tubos de aço & 415,00 & 569,50 \\
\hline Parafusos & 723,51 & 940,00 \\
\hline
\end{tabular}

O concreto utilizado foi proveniente da Empresa Lafarge Concreto S.A instalada em Belo Horizonte, MG. A concretagem foi realizada no Laboratório de Análise Experimental de Estruturas (LAEES) da UFMG. Foi utilizado concreto autoadensável com resistência média à compressão $\left(f_{c m}\right)$ de $42,08 \mathrm{MPa}$ e $41,87 \mathrm{MPa}$ para as séries $\mathrm{E}$ e $\mathrm{F}$, respectivamente. Esses valores foram obtidos por ensaio de caracterização realizado no LAEES. Nos subitens 2.2 e 2.3 são apresentados os resultados experimentais. A partir da curva força versus deslizamento relativo, os seguintes parâmetros foram obtidos:

- $P_{\text {máx }}$, definida como a força máxima obtida nas curvas;

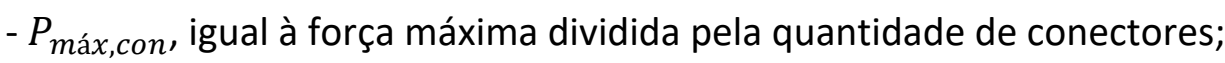

- $P_{R k}$, definida como a força resistente característica tomada como igual a 0,90 $P_{\text {máx }}$, com base no EN 1994-1-1:2004;

- $k_{s c}$, que é a rigidez secante medida a 70\% de $P_{R k}$, com base no EN 1994-1-1:2004.

\subsection{Resultados da série $E$}

Na Figura 5 são fornecidos os resultados da série $E$, constituída por dois modelos, HM1 e HM2. Observa-se que as curvas força versus deslizamento de ambos apresentaram um comportamento bem próximo no início da aplicação da força até alcançar um deslizamento relativo de aproximadamente $12 \mathrm{~mm}$. Para esse deslizamento, também se verifica que o modelo HM2 atingiu a sua força máxima $\left(P_{\text {máx }}\right)$. No entanto, o modelo HM1 alcançou $P_{\text {máx }}$ para um deslizamento relativo próximo de $25 \mathrm{~mm}$. Ambos os ensaios foram finalizados para um deslizamento relativo de aproximadamente $34 \mathrm{~mm}$.

A Tabela 2 apresenta a força máxima $\left(P_{\text {máx }}\right)$, a força máxima por conector $\left(P_{\text {máx,con }}\right)$, a força resistente característica $\left(P_{R k}\right)$ e a rigidez secante $\left(k_{s c}\right)$ dos modelos. Nota-se que os conectores apresentaram $k_{s c}$ inferior a $200 \mathrm{kN} / \mathrm{mm}$ e, dessa forma, podem ser classificados como flexíveis. 


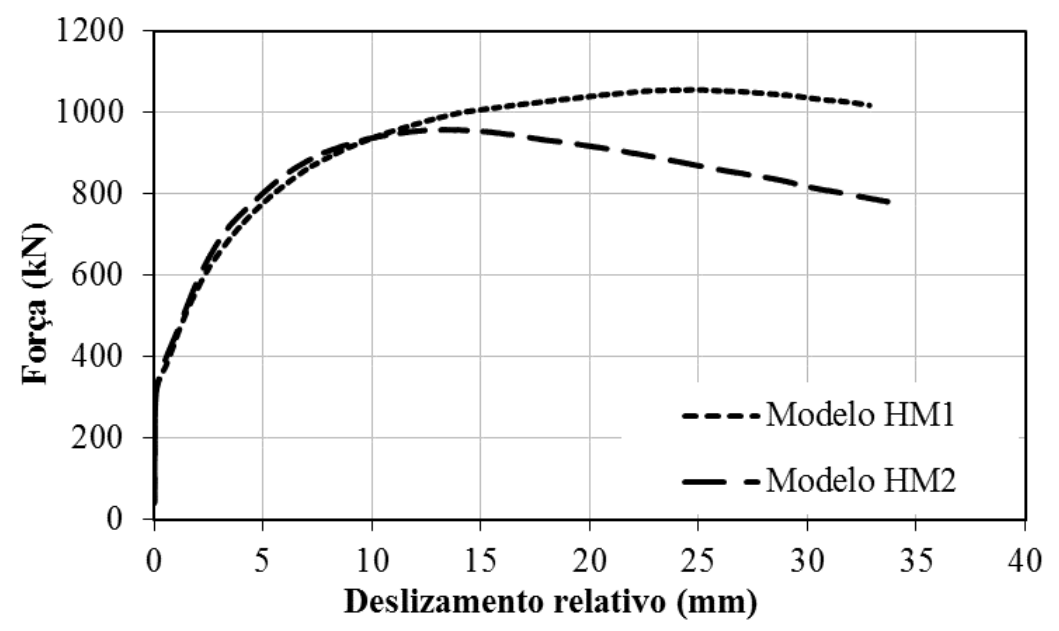

Figura 5 - Força versus deslizamento relativo dos modelos da série $\mathrm{E}$

A rigidez $k_{s c}$ dos conectores no modelo $\mathrm{HM} 2$ foi $33 \%$ superior a rigidez dos conectores no modelo HM1. Em relação a força máxima $P_{\text {máx }}$, observa-se que o modelo HM2 apresentou resultado apenas $10 \%$ inferior ao do modelo HM1, indicando que, em ambos os modelos, os conectores apresentaram valores de capacidade resistente próximos.

Tabela 2 - Valores de força e rigidez nos modelos da série $E$

\begin{tabular}{|c|c|c|c|c|}
\hline Modelo & $\boldsymbol{P}_{\text {máx }}$ & $\boldsymbol{P}_{\text {máx,con }}$ & $\boldsymbol{P}_{\boldsymbol{R k}}$ & $\boldsymbol{k}_{\boldsymbol{s c}}$ \\
\hline- & {$[\mathrm{kN}]$} & {$[\mathrm{kN}]$} & {$[\mathrm{kN}]$} & {$[\mathrm{kN} / \mathrm{mm}]$} \\
\hline HM1 & $1.057,34$ & 264,33 & 237,90 & 53,28 \\
\hline HM2 & 958,72 & 239,68 & 215,71 & 70,93 \\
\hline Média & $1.008,03$ & 252,01 & 226,80 & 62,10 \\
\hline
\end{tabular}

Na Figura 6 mostram os conectores e o núcleo de concreto após o ensaio. O tubo de aço foi cortado e os parafusos recolocados (em apenas um dos modelos) para possibilitar a visualização do núcleo de concreto. Santos et al. (2016) verificaram que os modelos iniciaram a perda de rigidez devido ao processo de fissuração do núcleo de concreto. À medida que os conectores foram acionados, o concreto situado abaixo dos parafusos (próximo ao tubo de aço) foi submetido a tensões de compressão elevadas, o que resultou em seu esmagamento. Com a intensificação do carregamento, houve a propagação das fissuras no núcleo de concreto próximo dos conectores, e estes apresentaram deformações plásticas. 
Houve maior concentração de fissuras no concreto em torno dos parafusos do nível superior (Figura 6). Supõe-se que esse fenômeno ocorreu porque o conector instalado nesse nível restringe o concreto acima dos conectores inferiores, contendo a fissuração no nível destes.
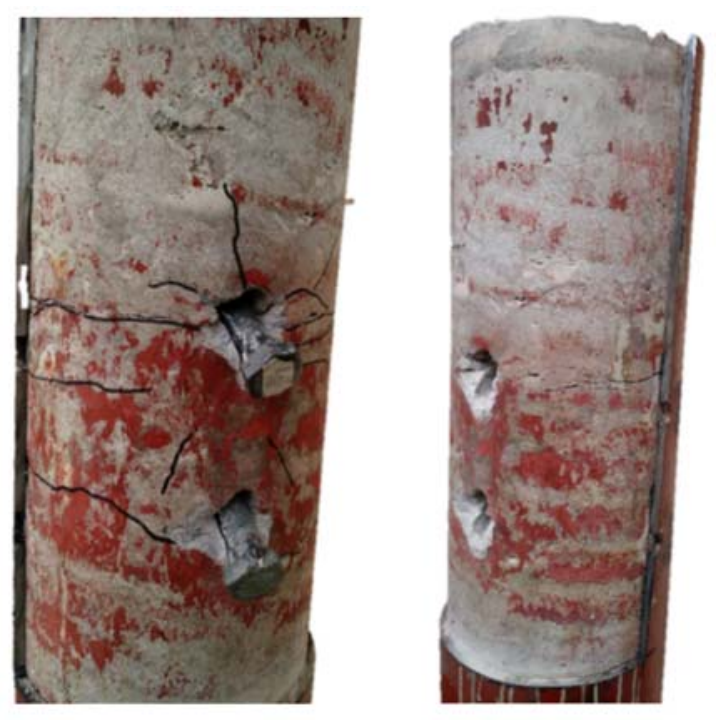

Figura 6 - Modelos da série E após o ensaio: visualização do núcleo de concreto e parafusos reposicionados após corte do tubo de aço

A Figura 7 apresenta a configuração deformada dos tubos e da cabeça dos conectores. Pode-se constatar a ocorrência de uma rotação significativa da cabeça dos conectores e de esmagamento local do tubo na região dos furos.
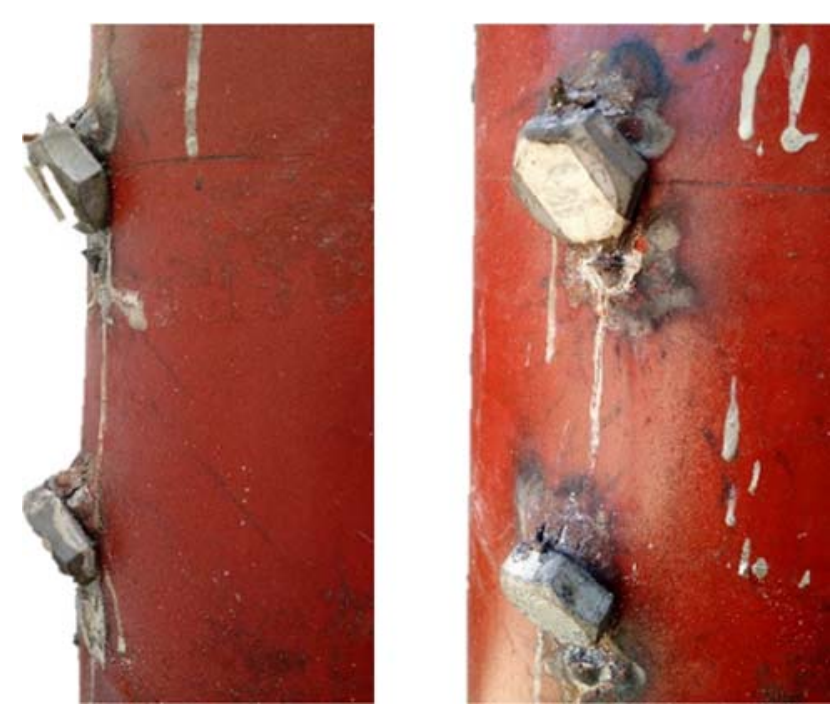

Figura 7 - Tubo de aço e cabeça dos conectores após o ensaio dos modelos da série $E$ 


\subsection{Resultados da série $F$}

A Figura 8 apresenta os resultados da série F. A curva força versus deslizamento do modelo HM3 fornece um valor de rigidez inicial inferior ao do modelo HM4. Dessa forma, o modelo HM3 apresentou forças inferiores até o deslizamento relativo de aproximadamente $6 \mathrm{~mm}$. Em seguida, o modelo HM3 mostrou possuir maior capacidade resistente até o término do ensaio.

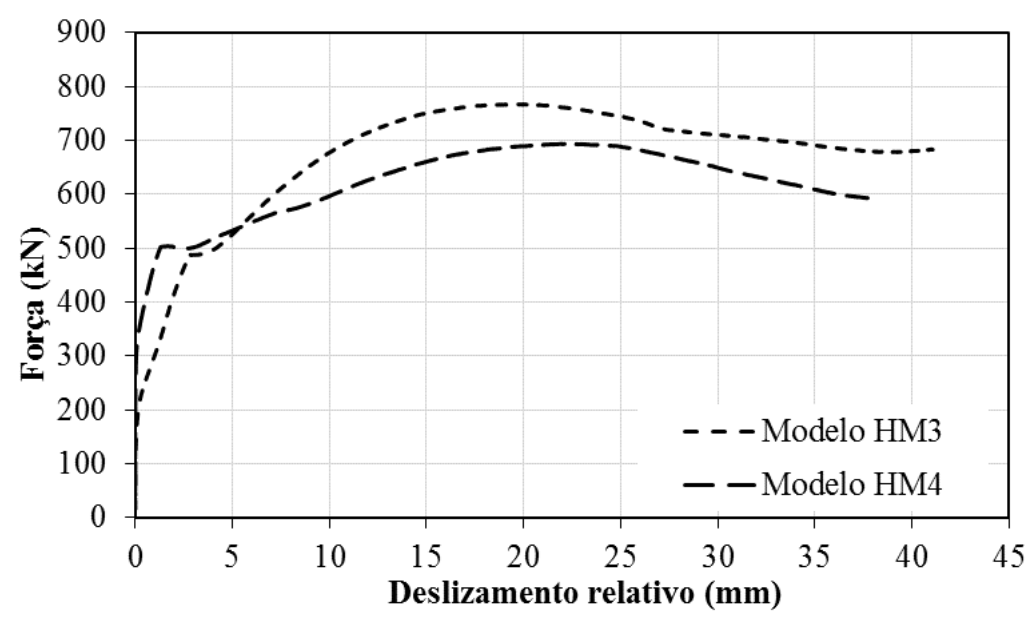

Figura 8 - Força versus deslizamento relativo dos modelos da série $F$

Na Tabela 3 observa-se que a rigidez $k_{s c}$ dos conectores do modelo HM4 foi consideravelmente elevada em relação a rigidez do modelo $\mathrm{HM} 3$, com uma razão de 3,35 . Também se observa que os conectores do modelo HM3 apresentaram uma capacidade resistente apenas $10 \%$ superior à do modelo HM4, ou seja, em ambos os modelos os conectores apresentaram capacidades resistentes próximas.

Tabela 3 - Valores de força e rigidez alcançados nos modelos da série $F$

\begin{tabular}{|c|c|c|c|c|}
\hline Modelo & $\boldsymbol{P}_{\text {máx }}$ & $\boldsymbol{P}_{\text {máx, con }}$ & $\boldsymbol{P}_{\boldsymbol{k} \boldsymbol{k}}$ & $\boldsymbol{k}_{\boldsymbol{s c}}$ \\
\hline- & {$[\mathrm{kN}]$} & {$[\mathrm{kN}]$} & {$[\mathrm{kN}]$} & {$[\mathrm{kN} / \mathrm{mm}]$} \\
\hline HM3 & 767,27 & 191,82 & 172,64 & 44,04 \\
\hline HM4 & 694,00 & 173,50 & 156,15 & 147,88 \\
\hline Média & 730,63 & 182,66 & 164,39 & 95,96 \\
\hline
\end{tabular}

Na Figura 9 tem-se um dos modelos da série $F$ após o ensaio. Com a elevação da força aplicada, ocorreu o giro dos parafusos e o escoamento do tubo de aço na região do furo em contato com o conector. 
A solicitação dos conectores provocou os seguintes fenômenos: o esmagamento do concreto na região abaixo dos parafusos e próxima ao tubo; e, a ruptura por tração do núcleo de concreto devido aos esforços de tração no nível da seção transversal que contém os conectores. (Santos et al., 2016b).
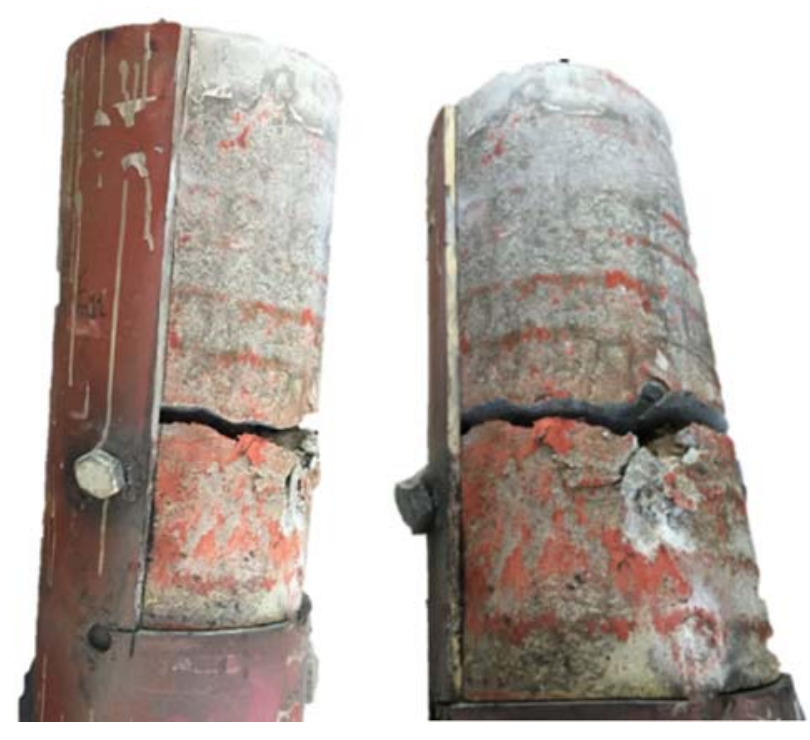

Figura 9 - Modelo da série F após o ensaio

\subsection{Comparação entre os resultados dos modelos das séries E e F}

A Tabela 4 apresenta os valores médios de força dos modelos das séries E e F. Verificase que a capacidade resistente dos conectores foi influenciada pela sua disposição em um ou dois níveis, apesar de o número total de conectores permanecer o mesmo.

Tabela 4 - Comparação entre os valores de força dos modelos das séries E e F

\begin{tabular}{|c|c|c|c|}
\hline séries & $\boldsymbol{P}_{\text {máx }}$ & $\boldsymbol{P}_{\text {máx,con }}$ & $\boldsymbol{P}_{\boldsymbol{R} \boldsymbol{k}}$ \\
\hline- & {$[\mathrm{kN}]$} & {$[\mathrm{kN}]$} & {$[\mathrm{kN}]$} \\
\hline $\mathrm{E}-2 \mathrm{~N}$ & $1.008,03$ & 252,01 & 226,80 \\
\hline $\mathrm{F}-1 \mathrm{~N}$ & 730,63 & 182,66 & 164,39 \\
\hline
\end{tabular}

A razão entre a força máxima $P_{\text {máx }}$ média dos modelos da série E (conectores em dois níveis) e a força média dos modelos da série $F$ (conectores em um nível) foi de 1,37. Supõe-se que um dos fatores determinantes na elevação da capacidade resistente dos modelos da série E seja o confinamento do concreto abaixo do conector, na região próxima à parede do tubo (Santos, 2017). Nos modelos da série E tem-se uma maior razão entre a área da seção transversal de concreto $\left(A_{c}\right)$ e a área solicitada pelo fuste 
do parafuso $\left(A_{1}\right)$, em uma mesma seção, o que proporciona uma maior tensão resistente do concreto devido ao confinamento. Essa justificativa também pode ser confirmada quando se faz uma comparação entre a Eq. (3) e a Eq. (9), correspondentes às tensões de compressão resistentes das normas ABNT NBR 16239:2013, que não leva em conta o confinamento proporcionado pelo tubo, e EN 1994-1-1:2004, que leva em conta esse efeito.

\section{Comparação dos Procedimentos de Projeto com os Modelos}

\section{Experimentais}

Na Tabela 5 são apresentados os resultados analíticos dos procedimentos de cálculo da norma ABNT NBR 16239:2013, de Starossek e Falah (2008) e de Van Long et al. (2015), sendo definidos pela Eq. (1), Eq. (7) e Eq. (9), respectivamente, considerando somente o mecanismo de falha no concreto. Na mesma tabela, esses resultados são comparados com os resultados obtidos na análise experimental pela razão $V_{R k} / P_{\text {máx. }}$ Os resultados apresentados correspondem ao valor da resistência de apenas um conector. Os coeficientes de ponderação da resistência foram considerados iguais a unidade. 0 coeficiente de comportamento do concreto $\gamma_{n}$ da Eq. (3) foi mantido como igual a 1,40.

Tabela 5 - Razão entre os resultados dos procedimentos analíticos de cálculo e dos ensaios considerando somente a falha no concreto

\begin{tabular}{|c|c|c|c|c|c|c|c|c|}
\hline \multicolumn{3}{|c|}{ Ensaios } & \multicolumn{6}{|c|}{ Procedimentos Analíticos } \\
\hline \multirow{2}{*}{\multicolumn{2}{|c|}{ Modelo }} & \multirow{2}{*}{$P_{\text {máx }}$} & \multicolumn{2}{|c|}{$\begin{array}{c}\text { ABNT NBR } \\
\text { 16239:2013 }\end{array}$} & \multicolumn{2}{|c|}{$\begin{array}{c}\text { Starossek e } \\
\text { Falah (2008) }\end{array}$} & \multicolumn{2}{|c|}{$\begin{array}{l}\text { Van Long } \\
\text { et al. (2015) }\end{array}$} \\
\hline & & & $V_{R k}$ & $\begin{array}{l}V_{R k} / \\
P_{\text {máx }}\end{array}$ & $V_{R k}$ & $\begin{array}{l}V_{R k} / \\
P_{\text {máx }}\end{array}$ & $V_{R k}$ & $\begin{array}{l}V_{R k} / \\
P_{\text {máx }}\end{array}$ \\
\hline & & {$[\mathrm{kN}]$} & {$[\mathrm{kN}]$} & - & {$[\mathrm{kN}]$} & - & {$[\mathrm{kN}]$} & - \\
\hline \multirow{2}{*}{ Série $E$} & HM1 & 264,33 & 99,26 & 0,38 & 112,02 & 0,42 & 288,52 & 1,09 \\
\hline & HM2 & 239,68 & 99,26 & 0,41 & 112,02 & 0,47 & 288,52 & 1,20 \\
\hline \multirow{2}{*}{ Série $F$} & HM3 & 191,82 & 98,77 & 0,51 & 112,58 & 0,59 & 376,36 & 1,96 \\
\hline & HM4 & 173,50 & 98,77 & 0,57 & 112,58 & 0,65 & 376,36 & 2,17 \\
\hline \multicolumn{3}{|c|}{ Média } & - & 0,47 & - & 0,53 & - & 1,61 \\
\hline \multicolumn{3}{|c|}{$\mathrm{COV}^{*}$} & - & 0,19 & - & 0,20 & - & 0,34 \\
\hline
\end{tabular}

Verifica-se que os procedimentos de cálculo da ABNT NBR 16239:2013 e de Starossek e Falah (2008) forneceram resultados próximos entre si, com os da norma brasileira 
inferiores em cerca de 13\%. Entretanto, esses resultados estão bem abaixo dos experimentais obtidos. Em ambos os modelos de cálculo, houve uma pequena melhora na aproximação aos valores experimentais nos modelos HM3 e HM4 da série F, que apresentam apenas um nível de instalação dos conectores.

O confinamento que o tubo de aço fornece ao núcleo de concreto apresenta-se como um fator significativo para predição da capacidade resistente dos conectores parafusos. Essa hipótese foi comentada no subitem 2.4, e aqui ela surge novamente quando se avalia os valores extremos das médias apresentadas na Tabela 5 , cujos valores são 0,47 e 1,61, para os modelos de cálculo da ABNT NBR 16239:2013 e Van-Long et al. (2015), respectivamente. O confinamento do concreto tende a ser maior ou menor, dependendo do fator de confinamento e da região parcialmente carregada considerada para o dimensionamento dos conectores. Esse comportamento está implícito nas equações correspondentes à falha do concreto desses modelos de cálculo. Ao avaliar a falha no concreto pela equação proposta por Van-Long et al. (2015), observa-se que esta foi a única que apresentou resultados não conservadores em relação aos experimentos, com uma média igual a 1,61 (Tabela 5).

A Tabela 6 compara os resultados considerando, agora, todos os possíveis modos de falha dos modelos de cálculo da norma ABNT NBR 1623:2013 utilizando-se a Eq. (1) a Eq. (2), de Starossek e Falah (2008) utilizando-se a Eq. (4) e Eq. (5), e de Van-Long et al. (2015) utilizando-se a Eq. (7) e Eq. (8).

Tabela 6 - Razão entre os resultados dos procedimentos analíticos de cálculo e dos ensaios considerando todos os modos de falha

\begin{tabular}{|c|c|c|c|c|c|c|c|c|c|}
\hline \multirow{3}{*}{ Modelo } & \multicolumn{9}{|c|}{ Procedimentos Analíticos } \\
\hline & \multicolumn{3}{|c|}{$\begin{array}{c}\text { ABNT NBR } \\
16239: 2013\end{array}$} & \multicolumn{3}{|c|}{$\begin{array}{c}\text { Starossek e } \\
\text { Falah (2008) }\end{array}$} & \multicolumn{3}{|c|}{$\begin{array}{c}\text { Van Long } \\
\text { et al. (2015) }\end{array}$} \\
\hline & $V_{R k}$ & Falha & $\begin{array}{l}V_{R k} / \\
P_{\text {máx }} \\
\end{array}$ & $V_{R k}$ & Falha & $\begin{array}{l}V_{R k} / \\
P_{\text {máx }} \\
\end{array}$ & $V_{R k}$ & Falha & $\begin{array}{l}V_{R K} / \\
P_{\text {máx }}\end{array}$ \\
\hline- & {$[\mathrm{kN}]$} & - & - & {$[\mathrm{kN}]$} & - & - & {$[\mathrm{kN}]$} & - & - \\
\hline HM1 & 99,26 & Concreto* & 0,38 & 112,02 & Concreto* & 0,42 & 160,69 & Cisalhamento** & 0,61 \\
\hline $\mathrm{HM} 2$ & 99,26 & Concreto* & 0,41 & 112,02 & Concreto* & 0,47 & 160,69 & Cisalhamento** & 0,67 \\
\hline HM3 & 98,77 & Concreto* & 0,51 & 112,58 & Concreto* & 0,59 & 160,69 & Cisalhamento** & 0,84 \\
\hline HM4 & 98,77 & Concreto* & 0,57 & 112,58 & Concreto* & 0,65 & 160,69 & Cisalhamento** & 0,93 \\
\hline Média & & - & 0,47 & & - & 0,53 & & - & 0,76 \\
\hline COV & & - & 0,19 & & - & 0,20 & & - & 0,19 \\
\hline
\end{tabular}

* Falha por esmagamento do concreto; ** Falha por cisalhamento do parafuso. 
No procedimento de cálculo da norma brasileira, a ruína em todos os modelos continuou ocorrendo por falha do concreto (a falha por cisalhamento do parafuso forneceu uma força resistente nominal igual a $107,13 \mathrm{kN}$ ), coerentemente com as observações experimentais. Entretanto, caso fossem considerados os coeficientes de ponderação das resistências (valores de cálculo), a média dos resultados da Tabela 6 seria alterada, podendo mudar, inclusive, o modo de falha predominante no modelo de cálculo.

O procedimento de cálculo de Starossek e Falah (2008) continuou mantendo como prevalecente a falha do concreto e proporcionou uma aproximação melhor com os ensaios do que o procedimento da norma brasileira e pior do que o modelo de VanLong et al. (2015).

Por fim, o procedimento de cálculo de Van-Long et al. (2015), quando comparado com os demais, respondeu com uma melhor aproximação frente aos resultados experimentais, com média das razões igual a 0,76 . No entanto, a falha predominante foi o cisalhamento nos parafusos, em ambas as séries, E e F, diferentemente do observado nos experimentos e, também, dos resultados dos demais procedimentos de cálculo.

Nas Figuras 10 e 11, respectivamente para as séries E e F, têm-se linhas horizontais indicando o valor de força máxima por conector obtida experimentalente $P_{\text {máx,con }}$ (média dos modelos de cada série) e, também, indicações dos valores previstos pelos procedimentos de cálculo (considerando todos os modos de falha previstos por cada modelo).

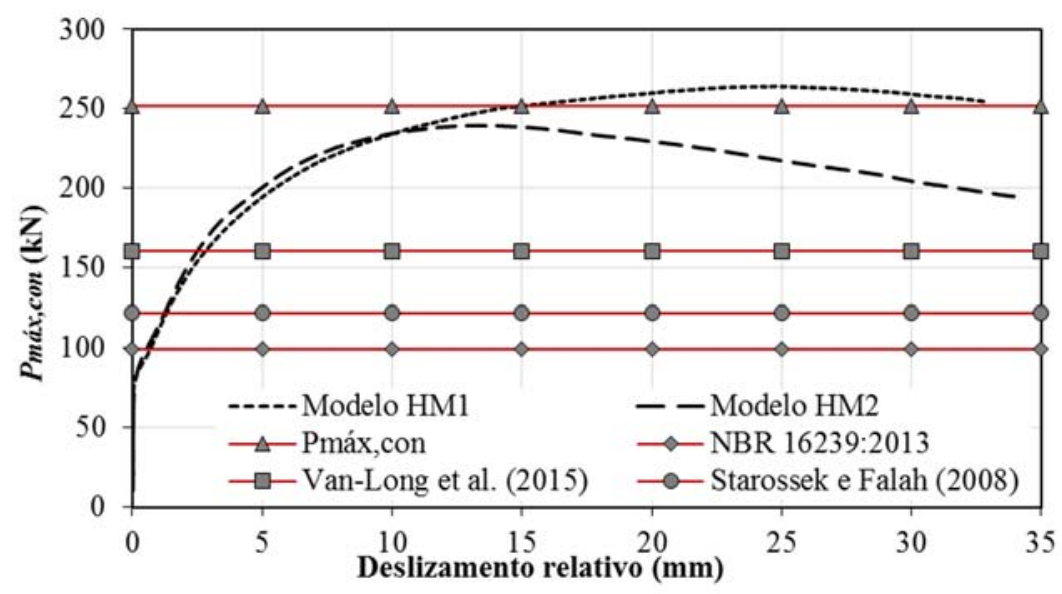

Figura 10 - Comparação entre as capacidades resistentes dos conectores da série $E$ 


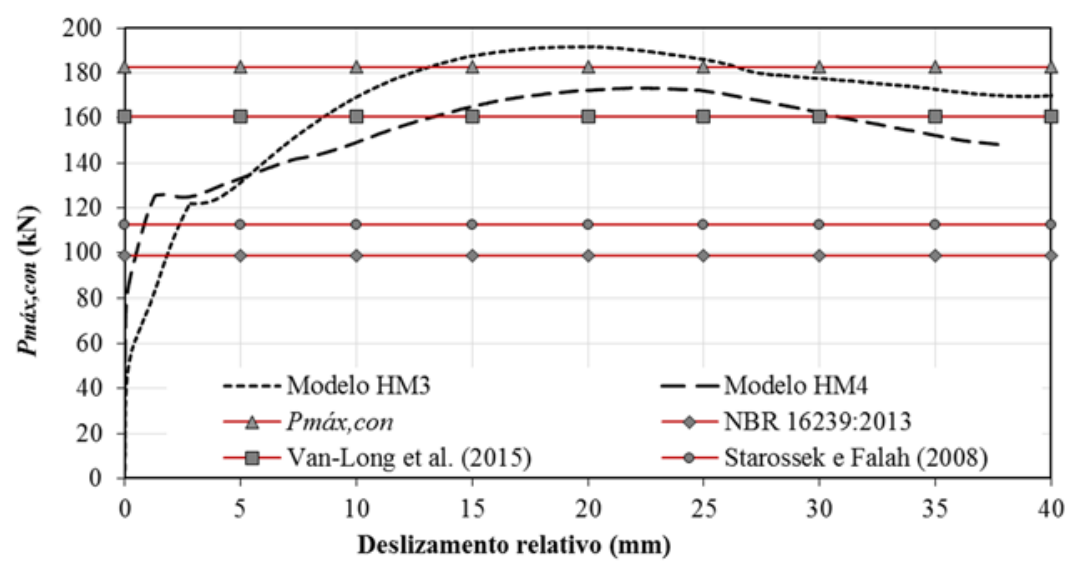

Figura 11 - Comparação das capacidades resistentes entre os conectores da série $F$

Os procedimentos de cálculo da ABNT NBR 16239:2013 e de Starossek e Falah (2008) forneceram uma razão igual a 0,88 e 0,87 para as séries E e $F(99,26 / 112,02$ e $98,77 / 112,58$, respectivamente). Isso sinaliza que esses procedimentos fornecem resultados bem próximos, como esperado, devido à semelhança das suas equações. Em todos os casos, o procedimento de cálculo da norma brasileira apresentou os menores resultados frente aos resultados experimentais, denotando seu conservadorismo. Em relação ao procedimento de Van-Long et al. (2015), apesar dos seus resultados finais serem mais próximos do experimental ao considerar apenas a falha no concreto (Tabela 5), observada nos experimentos, nota-se que esses resultados podem estar contra a segurança.

\section{Conclusões}

Este estudo buscou analisar os conectores parafusos como dispositivos de transferência de forças nos PMPC. A análise experimental foi constituída por duas séries que possibilitaram analisar o comportamento dos conectores de cisalhamento por meio da rigidez, força máxima e os modos de falha observados. Os resultados experimentais foram comparados com aqueles fornecidos pelos procedimentos de cálculo de Starossek e Falah (2008), Van-Long et al. (2015) e da ABNT NBR 16239:2013.

Nos experimentos realizados observou-se que o concreto foi esmagado localmente na região carregada abaixo do fuste dos parafusos. Em particular na série $F$ (conectores instalados em apenas um nível), o núcleo de concreto além de sofrer esmagamento localizado, foi submetido a esforços de tração pela ação das extremidades dos fustes 
dos parafusos. Essa ação contribuiu para a ruptura do núcleo de concreto por tração na seção transversal onde os conectores foram instalados.

Verificou-se que a disposição dos conectores na seção transversal dos PMPC foi um dos fatores determinantes na elevação de sua capacidade resistente, sendo esse ganho na ordem de $37 \%$, quando se comparou os resultados dos conectores instalados em dois níveis em relação aos que foram instalados em apenas um nível. Posto isso, na fase de projeto, torna-se indispensável avaliar a disposição dos conectores na seção transversal dos PMPC. Essa avaliação deve ser realizada de modo a se obter um nível maior de confinamento na região parcialmente carregada pelo fuste, elevando-se, assim, a capacidade resistente dos conectores para a transferência de forças.

Ao se analisar os procedimentos analíticos de cálculo, concluiu-se que o procedimento da ABNT NBR 16239:2013, na amostragem avaliada nesse estudo, conduziu a resultados significativamente inferiores aos obtidos experimentalmente. A razão entre os seus resultados e os experimentais apresentou a média mais baixa entre todos os procedimentos de cálculo, igual a 0,47.

O procedimento de cálculo de Starossek e Falah (2008) forneceu resultados próximos dos da norma brasileira, mas um pouco superiores a estes e que, portanto, se aproximaram mais dos experimentais, embora se mantivessem ainda bastante conservadores. A razão entre esses resultados e os experimentais teve uma média igual a 0,53, e um coeficiente de variação ligeiramente pior em relação aos demais, igual a 0,20 .

Por fim, observou-se que o procedimento de cálculo de Van-Long et al. (2015), mesmo sendo desenvolvido para parafusos passantes, respondeu com boa aproximação em relação aos resultados de ensaio quando foram avaliados todos os modos de falha. Todavia, ao se analisar a falha somente do concreto (observada experimentalmente), esse procedimento apresentou a pior aproximação, com uma média igual a 1,61, acima dos resultados dos ensaios.

Em linhas gerais, verifica-se que os resultados fornecidos pelos procedimentos de cálculo avaliados neste estudo ainda estão distantes da capacidade resistente dos conectores obtida experimentalmente. Além disso, no procedimento de Van-Long et al. 
(2015), o modo de falha prevalecente (falha do conector) não correspondeu ao observado experimentalmente (falha do concreto). Dessa forma, faz-se necessário estudos que busquem propor novos procedimentos cálculos capazes de retratar com melhor aproximação o comportamento dos conectores parafusos como dispositivos de transferência de força entre o tubo de aço e o núcleo de concreto em pilares tubulares preenchidos com concreto.

\section{Agradecimentos}

Os autores agradecem à FAPEMIG (Fundação de Amparo à Pesquisa do Estado de Minas Gerais), à CAPES (Coordenação de Aperfeiçoamento de Pessoal de Nível Superior), ao CNPq (Conselho Nacional de Desenvolvimento Científico e Tecnológico) e à Vallourec Tubos do Brasil S.A. pelo apoio.

\section{Referências bibliográficas}

ALMEIDA, Paulo Henrique Ferreira. Estudo numérico de um dispositivo de transferência de cargas em pilares mistos tubulares preenchidos com concreto. Programa de Pós-Graduação em Engenharia de Estruturas, Universidade Federal de Minas Gerais, Belo Horizonte, 2015. (Dissertação de Mestrado)

ATHIRA, T. J. Study on Structural Behaviour of Concrete Filled Steel Tubular Columns with and without Shear Connectores. International Journal of Innovative Research in Science, Engineering and Technology, v. 5, p. 13364-13372, July 2016.

ASSOCIAÇÃO BRASILEIRA DE NORMAS TÉCNICAS. NBR 8800:2008: Projeto de Estruturas de Aço e de Estruturas Mistas de Aço e Concreto de Edifícios. Rio de Janeiro, 2013.

ASSOCIAÇÃO BRASILEIRA DE NORMAS TÉCNICAS. NBR 16239:2013: Projeto de Estruturas de Aço e de Estruturas Mistas de Aço e Concreto de Edificações com Perfis Tubulares. Rio de Janeiro, 2013.

AMERICAN SOCIETY FOR TESTING AND MATERIALS. ASTM A325-10 ${ }^{\varepsilon 1}$. Standard Specification for Structural Bolts, Steels, Heat Treated, 120/105 ksi Minimum Tensile Strength. West Conshohocken, 2013.

BÄRTSCHI, R. Load-Bearing Behavior of Composite Beams in Low Degrees of Partial Shear Connection. Institute of Structural Engineering, ETH Zurich, 2005. (Phd Thesis)

CARDOSO, Hermano de Sousa. Estudo Teórico Experimental de Parafusos Utilizados como Dispositivos de Transferência de Carga em Pilares Mistos Tubulares Preenchidos com Concreto. Programa de Pós-Graduação em Engenharia de Estruturas, Universidade Federal de Minas Gerais, Belo Horizonte, 2014. (Dissertação de Mestrado) 
CARDOSO, Hermano de Sousa; CALDAS, Rodrigo Barreto; FAKURY, Ricardo Hallal. Método de ensaio de cisalhamento utilizado em Pilares Mistos Tubulares de Aço Preenchidos com Concreto. Revista Ciência \& Engenharia (Science \& Engineering Journal). Uberlândia, v. 15, p. 29-38, 2016.

EN 1994-1-1:2004, Eurocode 4: Design of Composite Steel and Concrete Structures, Part 1.1: General Rules and Rules for Buildings. European Committee for Standardization. Brussels, Belgium, 2004.

RIBEIRO NETO, J. G.; SARMANHO, A. M. Experimental analysis of a mechanical shear connector in concrete filled steel tube column. Ibracon Structures and Material Journal, v. 10, p. 592-625, 2017.

SANTOS, L. R.; CALDAS, R. B.; FAKURY, R. H.; RODRIGUES, F. C. Conectores parafusos instalados em dois níveis em pilares mistos preenchidos com concreto autoadensável. Congresso LatinoAmericano da Construção Metálica (CONSTRUMETAL), 7ạ ed., São Paulo, 2016a.

SANTOS, L. R.; CALDAS, R. B.; CARDOSO, H. S.; PRATES, J.A. Pilares mistos preenchidos com concreto autoadensável utilizando parafusos de alta resistência mecânica como conectores de cisalhamento. Anais do 58 Congresso Brasileiro do Concreto, CBC2016-IBRACON, Belo Horizonte, 2016b.

SANTOS, L. R. Análise numérica de conectores parafusos em pilares mistos circulares preenchidos com concreto. Programa de Pós-Graduação em Engenharia de Estruturas, Universidade Federal de Minas Gerais, Belo Horizonte, 2017. (Dissertação de Mestrado)

STAROSSEK, U.; FALAH, N. Force transfer in concrete-filled steel tube columns. Proceedings, 5th European Conference on Steel and Composite Structures - Eurosteel 2008, Graz, Austria, 2008.

VAN LONG, Hoang; JEAN PIERRE, Jaspart; DEMONCEAU, Jean-François. Extended end-plate to concrete-filled rectangular column joint using long bolts. Journal of Constructional Steel Research, Belgium, v. 113, p. 156-168, 2015.

YOUNES, Sherif M.; RAMADAN, Hazem M.; MOURAD, Sherif A. Stiffening of short small-size circular composite steel-concrete columns with shear connectors. Journal of Advanced Research, v. 7, p. 525-538, 2016. 